\title{
An Innovation in Corona Charging of Electrets
}

\author{
Rameshwar Nath ${ }^{1}$, Manju Mishra Patidar ${ }^{2}$, Nand Kishore Ghodke ${ }^{2}$, Sadan Chandra Das ${ }^{2}$. V Ganesan ${ }^{2}$ \\ ${ }^{1}$ Swami Vivekanand University) Sagar 470228, India ${ }^{2}$ UGC-DAE CSR Indore 452001, India
}

profrnath@gmail.com manjumishra.iuc@gmail.com nandkishor3105@ gmail.com scdascsr@gmail.com vganesancsr1@gmail.com

\begin{abstract}
An innovation in corona charging of electrets of polymer films has been adopted in locally designed and fabricated corona charging set up by replacing the regular high voltage DC power supply with a light, portable handy $3 \mathrm{~V}-6 \mathrm{~V}$ DC to DC $400 \mathrm{kV}$ boost step-up power module high voltage generator. Polymer film electrets of polyethylene transparency film have been corona charged using this innovative setup. The measurements were done and the results have been analyzed in the light of cited literature to show the effectiveness and advantage of the innovation.
\end{abstract}

Keywords - charge injection, corona discharge, high voltage, ions, polymers, surface potential decay

\section{.1. Introduction}

Electrets have been prepared using different methods for different electret types. [1] Corona charging of electrets can be done by depositing charge on the surface of electret material for example polymer film. Corona discharge processes provide charges in the form of ions and electrons for deposition on the surface of the material. Corona discharge is the electrical discharges taking place at or near atmospheric pressure. The corona is usually generated by a strong electric field associated with small diameter wires, needles, or sharp edges blades on an electrode and emphasizes one of the two aspects of the discharge, the ions produced or energetic electrons producing the plasma.

In the needle plate electrode configuration, the positive corona discharge starts with burst pulse corona and proceed to the streamer corona, glow corona and spark discharge while for negative corona in the same geometry the initial form is Trichel pulse corona followed by pulseless corona and spark discharge as the applied voltage increases.[2]

Making procedures in a conventional discharge device consists of an intense electric field in a neighborhood of biased corona point, usually tungsten wire, and grounded plate electrode to produce an electret of polymer film sample placed on the plate electrode. The electret is formed due to deposition and or penetration of the charge carriers produced in ionization of air at atmospheric pressure.

We have for the first time innovated the traditional corona charging set up by using a low cost (less than the US \$ 10) pulse width modulated DC- $3 \mathrm{~V}-6 \mathrm{~V}$ to DC $400 \mathrm{kV}$ boost stepup power module high voltage generator (Kits Guru-KG493 supplied by Amazon.in, Figure 1) in place of traditional high voltage power supplies which are quite expensive and cumbersome to use. This module uses the principle of the Tesla coil and is made of high voltage pulse output current. The device is a small production with a high-pressure science inverter transformer/booster based on

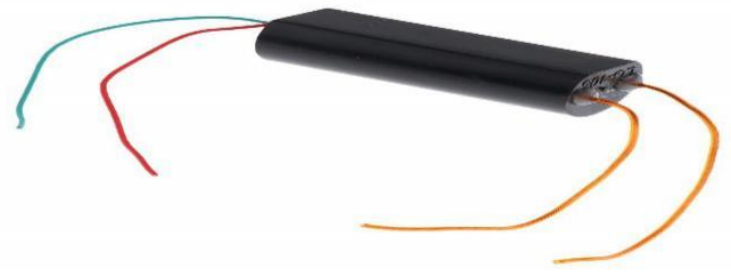

Fig.1 DC to DC High voltage module

technical review [3] and can be used as an ion generator high voltage source.

We have used our innovated easy handy portable set up to make corona charged electrets of commercially available transparencies films of polyethylene (PE) polymer. Our choice of material has been motivated to explore the possibility of using these electrets as a portable dosimeter being placed on various parts of the human body to monitor radiation exposure during radiotherapy treatment.

\section{Experimental Setup}

\section{A. Charging Setup)}

The corona discharge was generated using the locally designed and fabricated setup shown schematically in Figure 2 as the charging cycle. This setup uses a pulse width modulated DC 3- 6V to DC 400kV boost step-up transformer module (KG493) shown in Figure 1 as a high voltage source. This is powered by connecting the input terminals to a battery pack of $4 \times 1.5 \mathrm{~V}=6 \mathrm{~V}$ DC 4 A Duracells. The pulsed high voltage output is connected respectively to charging corona electrodes Anode and Cathode. The anode is a thin naked tungsten wire electrode of $350 \mathrm{~mm}$ length and $0.17 \mathrm{~mm}$ diameter. It is passed through a $2 \mathrm{ml}$ glass pipette for required safety in the use of high voltages. A circular brass disc of 50 $\mathrm{mm}$ diameter and thickness $04 \mathrm{~mm}$ forms the cathode which is earthed and

is connected to the cathode terminal of the output. Corona point electrode tungsten wire in the glass tube is held above the brass plate electrode by clamping it to stand with provision to change the height above the plate.

As a safety measure, the setup is provided with a switching circuit consisting of a momentary push to on switch to check the spark and a latching switch for continuous switching during charging duration. We have also made the provision 

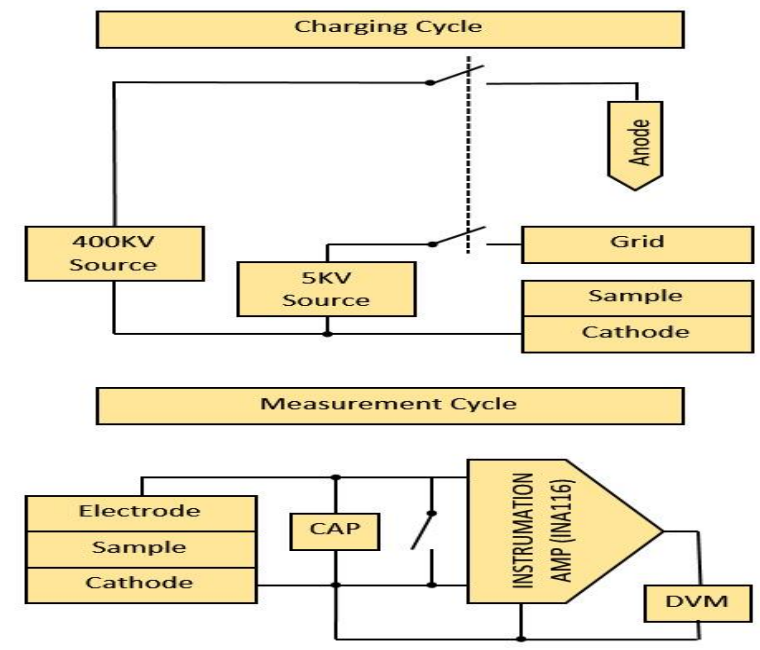

Fig. 2 Schematic diagram of Charging and Measuring setup

of a grid circuit using a similar high voltage module of lesser specifications and tested it for the possible use of a grid between point and plane electrode. However, for the present investigations of corona charging, we have used a dual-type electrode or a diode configuration. In this setup, the corona discharge current can be measured using an appropriate ammeter in series with the anode circuit.

\section{B Measuring Setup}

The measuring setup is a FET I/P High Impedance Preamplifier designed and fabricated locally using an ultralow input current (3fA), unit gain $(\mathrm{G}=1)$, and an instrumentation amplifier (INA116 from TI/BB ). The circuit is biased with DC batteries of $9 \mathrm{~V}$. The schematic diagram of the measuring setup is shown as a measuring cycle in Figure 2. The standard capacitor of $1 \mu \mathrm{F}$ used in the setup is equipped with a push to on switch across it to reset before every measurement. The surface charge on the surface of the sample electret is measured as surface potential in volts by transferring the charge to the standard capacitor of $1 \mu \mathrm{F}$ and measuring the potential developed in volts. The surface potential can be converted to charge in Coulomb according to relation $\mathrm{Q}=\mathrm{CV}$. The probe used in charge transfer is a solid brass disc of $28 \mathrm{~mm}$ diameter with a knob.

\section{Experimental Procedure}

We have used the following parameters and procedures for our experimental studies.

- DC high voltage was kept constant keeping input voltage fixed at $6 \mathrm{~V}$ using 4 cells of $1.5 \mathrm{~V}$.

- Corona point electrode was kept positive and the plate electrode was earthed throughout our measurements.

- The polymer sample surface in contact with the plate electrode was not metal coated.

- The sample surface facing the point electrode has been termed as Top surface and the surface in contact with the plate electrode is termed as Bottom surface.

- The surface potential measurements have been done on both the surfaces of corona charged electrets.
- Top surface measurement has been done immediately after the expiry of the charging time while the bottom surface measurement could be done after 5 seconds of the top surface measurement due to manually reversing the surface using plastic forceps.

- The top surface has been marked to distinguish with the bottom surface.

- The measured values have been manually recorded.

- Circular disc samples of diameter $30 \mathrm{~mm}$ have been cut out of polymer polyethylene (PE) transparencies sheets of $50 \mu \mathrm{m}$ thickness.

- Four series of 4 electrets each have been made by keeping the point to plate electrode distance fixed at $5 \mathrm{~mm}, 10 \mathrm{~mm}, 15 \mathrm{~mm}$, and $20 \mathrm{~mm}$ varying the charging time as $5 \mathrm{~s}, 10 \mathrm{~s}, 15 \mathrm{~s}$, and $20 \mathrm{~s}$ (seconds) for each series at a fixed distance.

- The surface potential measured immediately after charging on the Top and The Bottom surfaces are recorded as initial surface potential.

- The surface potential decay(SPD) is recorded by measuring the surface potential on both the surfaces respectively after the $30 \mathrm{~s}, 60 \mathrm{~s}, 90 \mathrm{~s}, 120 \mathrm{~s}, 150 \mathrm{~s}, 180 \mathrm{~s}$, and 210 s(seconds) of the initial measurement.

- The results of experimental studies have been analyzed in light of the possible available literature on the subject to understand the effectiveness of the innovation in corona charging of polymer electrets in the air under ambient conditions.

\section{Results and Discussions}

We have prepared four series of polyethylene (PE ) polymer electrets using our experimental setup for corona charging by keeping point to plate electrode distance fixed at $5 \mathrm{~mm}, 10 \mathrm{~mm}, 15 \mathrm{~mm}$, and $20 \mathrm{~mm}$ respectively for each series. We have varied corona charging time as 5 seconds (s), 10s, 15s, and 20s for four electrets in each series. We have made measurements on each electret for surface potential decay after every $30 \mathrm{~s}$ up to 210s.

Experimental results obtained have been analyzed in terms of various parameters to understand the consistency of used innovation in corona charging of electrets and their properties.

The surface potential developed on both the top and bottom surfaces of the sample has been measured and the results are shown in Figure 3. Figure 3(a) shows the surface potential decay measurement as a function of the charging time of $5 \mathrm{~s} .10 \mathrm{~s}, 15 \mathrm{~s}$, and $20 \mathrm{~s}$ for the four series. The top surface facing the point electrode of each electret shows positive potential while the bottom surface in contact with the plate electrode as also 
a) Surface Potential Decay
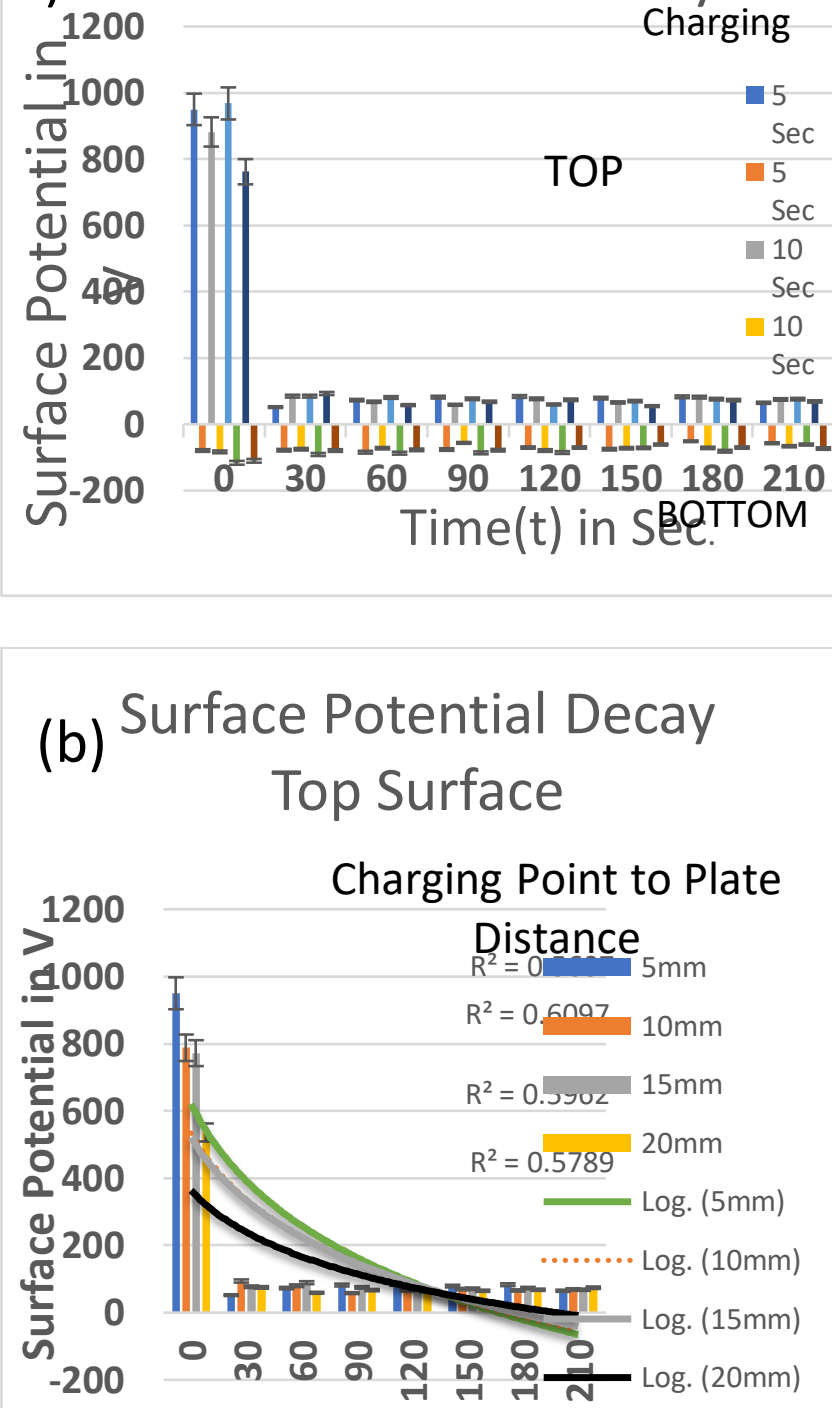

Time in Sec.

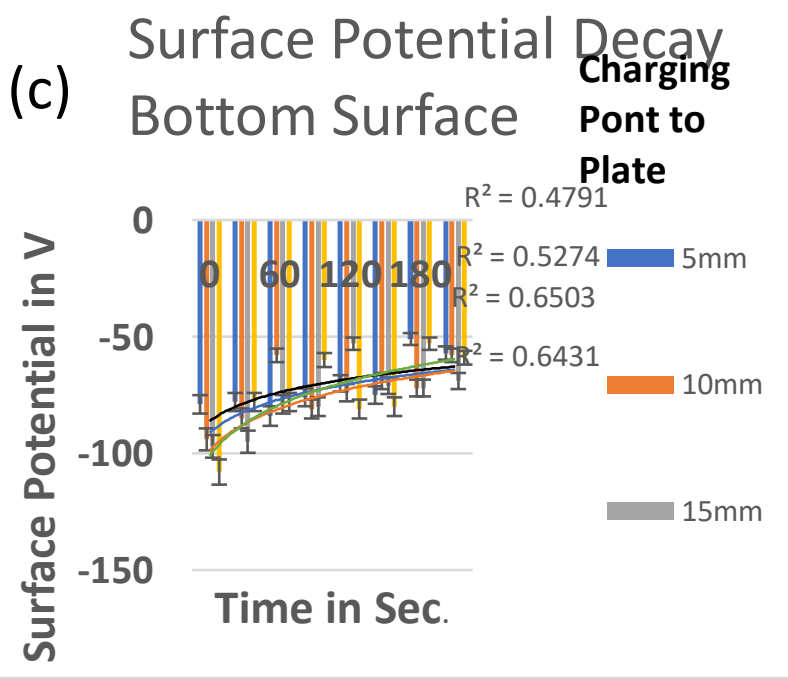

Fig. 3 Surface potential decay as a function of charging time for top and bottom surfaces (a), as a function of electrode distance top surface (b surface and bottom $(c)$ reported by Leandra P. Santos et al [4] and Chen's group $[5,6,7]$. Polymer surfaces under corona charging acquire high electrostatic potential due to the deposition of positive and negative ions moving through the atmosphere under the influence of the electric field. $[8,9,10]$. During corona charging, it is reported that polyethylene bottom surface negative charge is due to the injection of the electron from the cathode and holes from the anode according to Schottky Mechanism [7]. Contributions from the ions derived from a sample polymer as the charge carriers in contact and tribocharging have also been reported [11,12,13,14,15] Surface charging process may also take place within the space charge threshold established by Montanari et al [16,17].

In Figure 3(b) and 3(c), we found that the best-fit trendlines show the decreasing trend of the surface potential with decay time for different electrode distances. A strong dependence of the deposited charge on the surface potential decay evolution is indicated in a rapid decrease of charge on the sample surface To study this potential deposited effect on the sample surface we normalized the initial surface potential $\mathrm{V}_{0}$ as shown in Figures 4(a) and 4(b) respectively for top and bottom surfaces. The nature of curves shows the existence of field-effect on the sample which may indicate that the phenomenon of electron trapping, electron acceleration depends on the field. An electron hopping from the level of the surface trap into the bulk is charge injection dependent on the field. This can further assist the

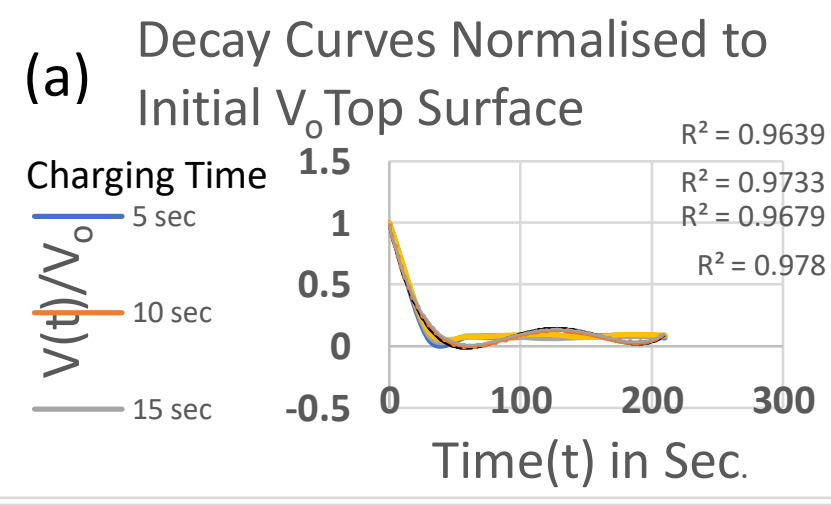

(b) Decay Curves Normalised to

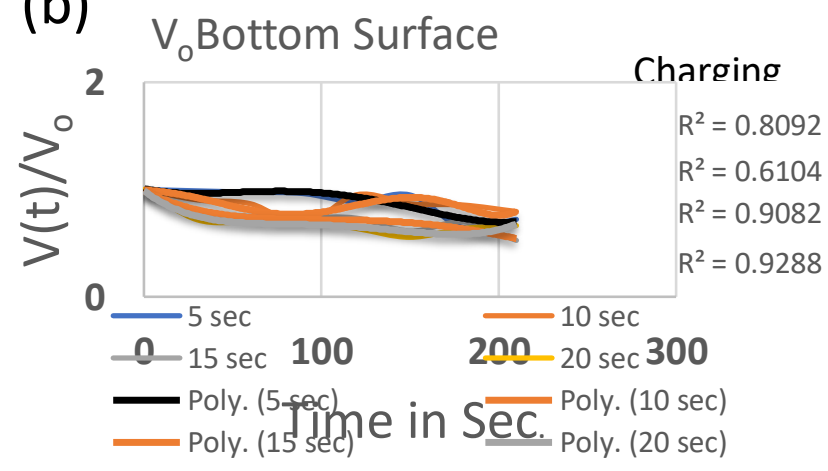

Fig. 4 Decay curves normalized to $\mathrm{V}_{0}$ top surface (a) and bottom surface (b) 


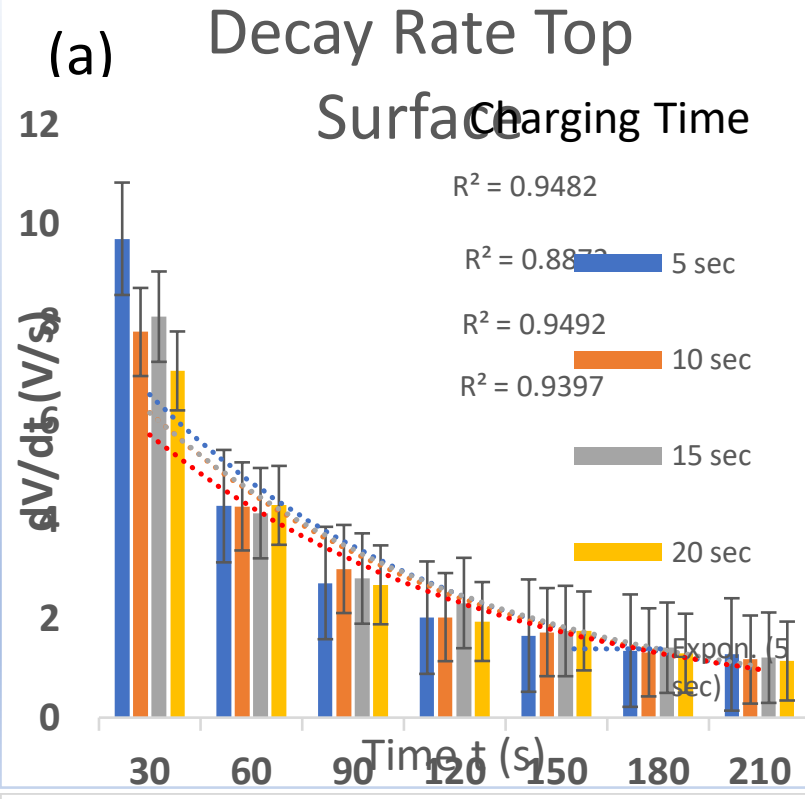

\section{(b) Decay Rate Bottom Surface}

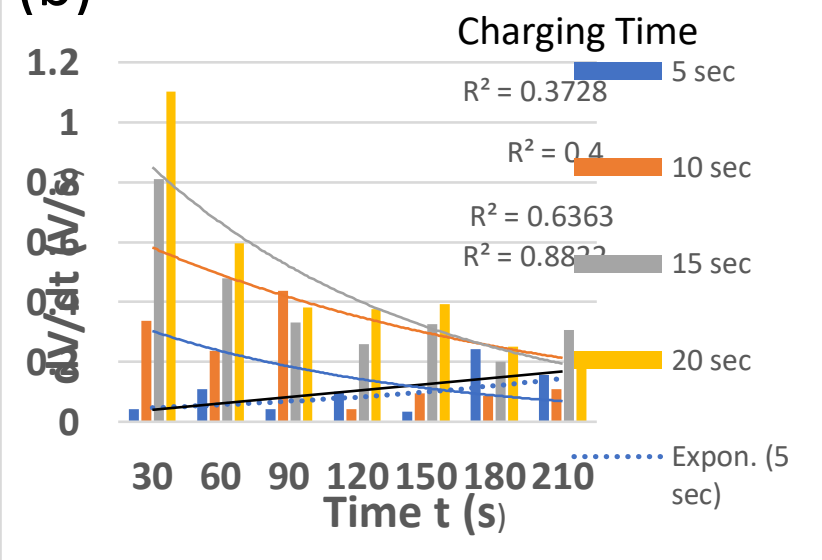

Fig. 5. Decay Rate top surface (a) and bottom surface (b)

electron to hop and then accelerate into the sample moving under their electric field towards the grounded plate electrode.[18] In our case point to plate configuration with the pointed tip of the tungsten wire may produce a high electric field and may inject a charge into the dielectric possibly also into the deep traps.[19,20,21,22].

Decay rate calculations from Figure 3(b) and 3(c) have been represented in Figure 5(a) and 5(b) respectively for top and bottom surfaces. The best fit trend is found to be exponentially showing a high initial growth rate followed by a slower value. The strong decrease initially suggests the deposition of injected charges under the field effect created by their charge. This leads to a crossover phenomenon since the injected charge is dependent on the quantity of the deposited charge. Local field dependence of the injected charges leads them to the sample bottom surface. Molinie [23,24,25.] has shown in his work that the material polarization mechanism gives an absorption current

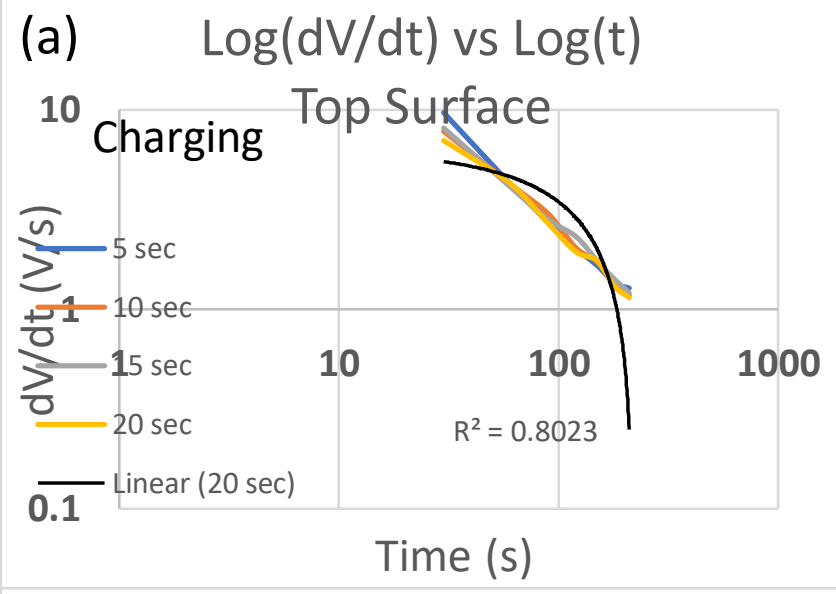

\section{$\log (d V / d t)$ vs $\log (t)$}

(b) Bottom Surface

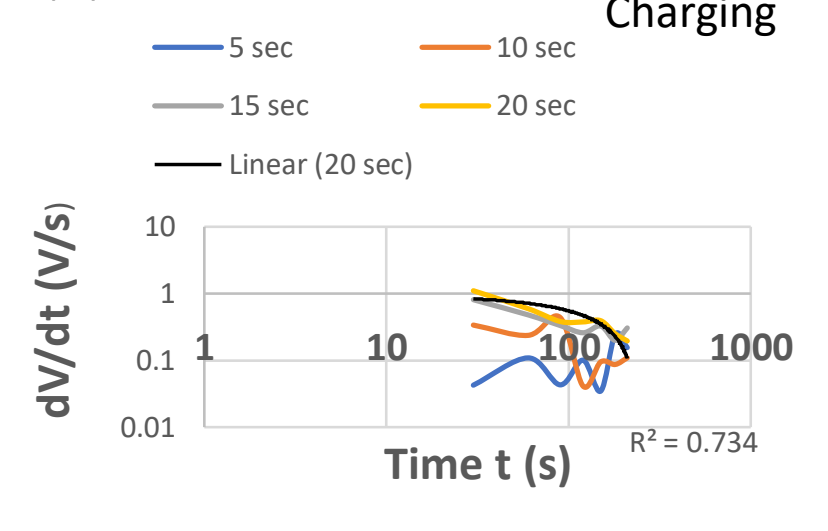

Fig. 6 Log - Log Plot of Decay Rate.

according to Curie-von Schweidl law which can be linked to surface potential decay rate $\mathrm{dV} / \mathrm{dt}$. Therefore, the decay rate $\mathrm{dV} / \mathrm{dt}$ can be conveniently used to understand the conduction intervening nature at charge deposition.

We have displayed the data of Figures 5(a) and 5(b) on the $\log$-Log plot showing the variation of $\log (\mathrm{dV} / \mathrm{dt})$ with $\log (\mathrm{t})$ in Figures 6(a) and 6(b). We note that the linear best fit on the curves is a curve having two straight-line slopes indicating two regimes of surface potential decay separated by a transit time to be determined by the intersection point of the two slopes[26,27]. Finally, we could measure the retained charge on the corona charged electret surfaces after a lapse of 136 days as due to COVID- 19, lockdown and other restrictions in India we could not monitor the retained charge on daily weekly or monthly basis. Never the less the Figure 7, showing the results of measurement, reveal the successful formation of electrets of polymer films using our innovative setup for corona charging of electrets. 
Retained Surface Charge After 130 Days ToP

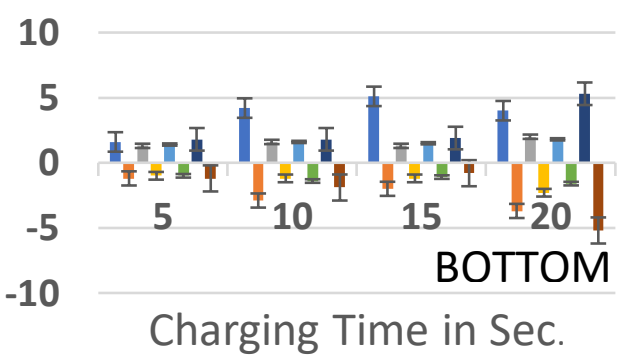

Point to Plate

$\square 5 \mathrm{~mm} \square 10 \mathrm{~mm} \square \square 15 \mathrm{~mm} \square \square 20 \mathrm{~mm}$

Fig. 7 Retained Surface charge on both the electret surfaces after 136 days.

\section{Conclusions}

We have successfully demonstrated the use of our handy innovation of using DC $3 \mathrm{~V}-6 \mathrm{~V}$ to DC $400 \mathrm{kV}$ booster step-up power module high voltage generator in corona charging of polymer film electrets. Our results of measurements on the electret have been successfully analyzed and discussed in the light of the work cited in the literature showing the effectiveness and advantage of our innovation. We plan to optimize the setup and the method according to the need of the applications.

\section{Acknowledgments}

The authors are thankful to the UGC-DAE CSR, Indore, India Director Dr. A. K. Sinha, Scientist H Dr. N. P. Lalla for fruitful interaction and to the inmates of LT Laboratory N. Patil, M. Gangrade for assistance. R. Nath also thanks SVN University, Sagar, India Managing Director Dr. Anil Tiwari for Institutional Support and Assistant Professor Dr. R. K. Rai for assistance in the manual recording of experimental observations.

\section{References}

[1] G.M. Sessler, Electrets, $2^{\text {nd }}$ ed. Springer- Verlag, New York, 1987

[2] Jen-Shih Chang, Phil A. Lawless, and Toshiaki Yamamoto, "Corona Discharge Processes", IEEE TRANSACTIONS ON PLASMA SCIENCE, VOL. 19, NO. 6, DECEMBER 1991 pp 1152-1166 ]

[3] Mojtaba Forouzesh, YamP. Siwakoti, Saman A. Gorji, Frede Blaabjerg, and Brad Lehman, ” Step-Up DC-DC Converters: A Comprehensive Review of Voltage-Boosting Techniques, Topologies, and Applications", IEEE TRANSACTIONS ON POWER ELECTRONICS, VOL. 32, NO. 12, DECEMBER 2017 pp9143-9178

[4] Leandra P. Santos, Juliana S. Bernardes, and Fernando Galembeck Corona-Treated Polyethylene Films Are Macroscopic Charge Bilayers", Langmuir 2013, 29, 892-901

[5] Zhiqiang, X.; Zhang, L. W.; Chen, G. Decay of Electric Charge on Corona Charged Polyethylene. J. Phys. D: Appl. Phys. 2007, 40, 7085-7089

[6] Chen, G.; Xu, Z.; Zhang, L. W. Measurement of the Surface Potential Decay of Corona-Charged Polymer Films Using the Pulsed Electroacoustic Method. Meas. Sci. Technol. 2007, 18, 1453-1458.

[7] Chen, G. A New Model for Surface Potential Decay of Corona-
Charged Polymers. J. Phys. D: Appl. Phys 2010, 43, 055405 (7pp).

[8] Giacometti, J. A.; Oliveira, O. N. Corona Charging of Polymers. IEEE Trans. Electr. Insul. 1992, 27, 924-943.

[9] Giacometti, J. A.; Fedosov, S.; Costa, M. M. Corona Charging of Po lymers: Recents Advances on Constant Current Charging. Braz. J. Phys. 1999, 29, 269-279.

[10] Ramachandran, N.; Jaroszeski, M.; Hoff, A. M. Molecular Delivery to Cells Facilitated by Corona Ion Deposition. IEEE Trans. Nanobiosci. 2008, 7, 233-239.

[11] McCarty, L. S.; Winkleman, A.; Whitesides, G. M. Ionic Electrets: Electrostatic Charging of Surfaces by Transferring Mobile Ions upon Contact. J. Am. Chem. Soc. 2007, 129, 4075-4088.

[12] Thomas, S. W.; Vella, S. J.; Kaufman, G. K.; Whitesides, G. M. Patterns of Electrostatic Charge and Discharge in Contact Electrification. Angew. Chem., Int. Ed. 2008, 47, 6654-6656.

[13] McCarty, L. S.; Whitesides, G. M. Electrostatic Charging due to Separation of Ions at Interfaces: Contact Electrification of Ionic Electrets. Angew. Chem., Int. Ed. 2008, 47, 2188-2207

[14] Baytekin, H. T.; Patashinski, A. Z.; Branicki, M.; Baytekin, B.; Soh, S.; Grzybowski, B. A. The Mosaic of Surface Charge in Contact Electrification. Science 2011, 333, 308-312.

[15] Burgo, T. A. L.; Ducati, T. R. D.; Francisco, K. R.; Clinckspoor, K. J.; Galembeck, F.; Galembeck, S. E. Triboelectricity:Macroscopic Charge Patterns Formed by Self-Arraying Ions on Polymer Surfaces. Langmuir 2012, 28, 7407-7416.

[16] Montanari, G. C. Dielectric Material Properties Investigated through Space Charge Measurements. IEEE Trans. Dielectr. Electr. Insul. 2004, 11, 56-64.

[17] Montanari, G. C.; Morshuis, P. H. F. Space Charge Phenomenology in Polymeric Insulating Materials. IEEE Trans. Dielectr. Electr. Insul. 2005, 12, 754-765.

[18]. K Smili., L Herous. "Corona Charging and Charge Decay on Polyethylene Terephthalate Films (PET)", in Electrotehnica, Electronica, Automatica (EEA), 2019, vol. 67, no. 3, pp. 81-90, ISSN 1582-5175.

[19] H.V. Segger, "Detection of surface and bulk traps", J.Appl. Phys. 52(6). pp. 4086-4089, 1981.

[20] D.M. Taylor, T.J. Lewis, "Electrical conduction in polyethylene terephthalate and polyethylene films", Phys. D4, p. 1346, 1971.

[21] D.M. Taylor, "Electron-beam charging of polyethylene terephthalate films", J .phys. D .Appl .phys, Vol. 9. pp. 2269-2279, 1976.

[22] M. Keiichi, M. Teda "Current peaks observed in polyethylene terephthalate films with linearly increasing voltage", Jap. Jour. Of. Appl. Phys, Vol.19, N6, pp. 1067-1071, 1980.

[23] P. Molinié, "Charge injection in corona-charged polymeric films: potential decay and current measurements", J. Electrostatics, Vol. 45, pp. 265-273, 1999.

[24] P. Molinie, P. Llovera, "Surface potential measurement: implementation and interpretation", 8th int. conf. DMM. Edinburgh, 2000.

[25] P. Molinie, M. Goldman, J. Gattelat, "Surface Potential decay on corona-charged epoxy samples due to the po larization process" J. Phys. D, vol.28, pp. 1601 1610,1995

[26] H. V. Berlepsch, "Interpretation of surface potential kinetics in HDPE by a trapping model", J. Phys. D., vol. 18, pp. 11551170,1985

[27] J. Bigarré, P. Hourquobie, L. doucet, J. Matallana, "New setup of surface potential decay", 3 rd Conf. French Society of Electrostatics, Toulouse, France, 2002. 